\title{
MAGNETIC EXCITATIONS IN THULIUM METAL
}

\author{
J. A. Fernandez-Baca, R. M. Nicklow \\ Solid State Division, Oak Ridge National Laboratory, Oak Ridge, TN 37331-6993
}

J. J. Rhyne,

National Institute of Standards and Technology, Gaithersburg, MD 20599

\begin{abstract}
We have performed inelastic neutron scattering measurements on a single crystal specimen of $\operatorname{Tm}$ at wavevectors $\vec{\kappa}=(1,1, \zeta)$ and $(0,0,2+\zeta)(\zeta=0, \ldots, 1)$. Most of the measurements have been made at $T=5 K$, where $T m$ exhibits a seven layer ferrimagneticantiphase-domain structure ( four moments up, parallel to the c-axis, followed by three moments down). At this temperature the excitation spectra consist of three peaks. The two lower energy excitations have been identified as originating from magneto-vibrational scattering from the TA phonon, while the higher energy excitation is magnetic and exhibits only a weak dispersion (between 8.3 and $9.6 \mathrm{meV}$ ). At $T=50 K$, a tempe ature at which the system exhibits a c-axis sinusoidally modulated structure, the magnetic mode shows significant softening and broadening. The magneto-vibrational scattering vanishes above the Nèel temperature $\left(T_{N}=58.5 K\right)$ while the magnetic mode persists at least up to $T=70 \mathrm{~K}$. These results suggest that the Hamiltonian in this system is dominated by the crystal-field-anisotropy energy, and that the exchange interaction is relatively weak.
\end{abstract}

PACS: 75.30.Ds, 75.50.Gg

\section{DISCLAIMER}

\begin{abstract}
This report was prepared as an account of work sponsored by an agency of the United States Government. Neither the United States Gnvernment nor any agency thereof, nor any of their employees, makes any warranty, express or implied, or assumes any legal liability or responsibility for the accuracy, completeness, or usefulness of any information, apparatus, product, or process disclosed, or represents that its use would not infringe privately owned rights. Reference herein to any specific commercial product, process, or service by trade name, trademark, manufacturer, or otherwise does not necessarily constitute or imply its endorsement, recommendation, or favoring by the United States Government or any agency thereof. The views and opinions of authors expressed herein do not necessarily state or reflect those of the United States Government or any agency thereof,
\end{abstract}

\footnotetext{
- The suemutted manuscript has beon authored by a contractor of the US. Government under contract No OE-ACO5-84OR2 1400 . Accordingly. the US. Governmont retains a nomeaclusive. rovaliy fore ireense to pubtish or repraduce

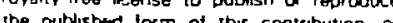
the Dublished lorm of this contribution, of athow others to do so. for U $\mathbf{5}$ Governman
} 


\section{INTRODLCTION}

In the last 25 years very detailed studies of the magnetic excitations of most of the heary rare earth elements have been published. ${ }^{1}$ These studies, which were performed utilizing neutron scattering techniques, have greatly contributed to our present understanding of the interactions that determine the complicated magnetic structures found in these systems. The study of the magnetic excitations in Tm, on the other hand, has been hampered by the lack of large high-quality crystals of this element and it is only in very recent years that efforts to perform this study have been undertaken. ${ }^{2-3}$ In this paper we report our progress in this study.

Thulium is the last of the magnetic heavy rare elements. It has an hcp structure and exhibits a very unusual series of magnetic structures. ${ }^{4}$ Below the Nèel temperature $T_{.}$the magnetic moments order in a c-axis sinusoidally modulated phase. Upon cooling the sinusoidal structure gradually squares up and below a temperature $T_{c}$ it develops a ferrimagnetic component and the system locks in a seven-layer ferrimagnetic antiphaseciomain structure ( four inoments up, parallel to the c-axis, followed by three moments down).

The nature of the magnetic excitations in the heavy rare earth elements is determined by the Hamiltonian ${ }^{\mathrm{I}}$ :

$$
\mathcal{H}=\mathcal{H}_{\text {ex }}+\mathcal{H}_{c f}
$$

In this equation $\mathcal{H}_{e x}$ is the exchange energy, which is due to a long-range oscillatory exchange interaction between highly localized $4 f$ moments. It is predominantely isotropic and has a Heisenberg form. The second term, $\mathcal{H}_{c f}$, is the crystai-field anisotropy energy. Other sources of anisotropy (magnetoelastic energy, etc) are neglected in this Hamiltonian. It is frequently assumed that in the heavy rare earth elements $\mathcal{H}_{c f} \quad<\mathcal{H}_{\text {ex }}$ and that the only effect of the crystal-field anisotropy is to impose a preferred direction of the magnetization. Th- energy levels under this assumption are equally-spaced $J_{z}$ levels and the elementary excitations of the system are spin waves, or collective propagating excitations from the $\left\langle J_{z}\right\rangle=J$ ground state. In this model the dispersion relation can be obtained by means of Holstein-Primakoff-type transformations followed by the proper diagonalization of the transformed Hamiltonian. This approach has been used succesfully to describe the magnetic excitations of $\mathrm{Gd}, \mathrm{Tb}, \mathrm{Dy}$ and Ho. ${ }^{1}$

When $\mathcal{H}_{c f} \approx \mathcal{H}_{e x}$, on the other hand, the energy diagram of the system does not consist of equally-spaced $J_{z}$ levels and Holstein-Primakoff-type transformations are not applicable. In this case a transformation that properly includes the effect of the crystal field on the energy diagram must be carried out. This has been performed by Lingardd and Lingård and Cooke ${ }^{5}$ to obtain the dispersion relation of Er. In the case where $\mathcal{H}_{c f}>\mathcal{H}_{e x}$ the energy diagram consists essentially of crystal-field levels and the exchange imposes a small dispersion on the excitations. In the extreme case where $\mathcal{H}_{e x}=0$ the energy diagram consists only of crystal-field levels. The study of the magnetic excitations of $\mathrm{Tm}$ is of interest because this is a system in which the crystal-field anisotropy is apceted to be at least comparable to the exchange interaction. 
The sample used in our experiments was a high-quality single crystal of Tm prepared at Ames Laboratories, Ames, Iowa. The neutron scattering measurements were performed with a con . ntional triple axis spectrometer at the National Institute of Standards and Technology (formerly National Bureau of Standards) reactor. The measurements were pe-formed in the constant scattering wavevector $\vec{r}$ mode at $\vec{\kappa}=(1,1, \zeta)$ and $(0,0,2+\zeta)$ (for $\zeta=0, \ldots, 1$ ) with a fixed final neutron energy $E_{f}=14.8 \mathrm{meV}$. A pyrolitic graphite filter was used in order to eliminate higher-order wavelength contaminations. Soller-slit collimators were utilized to obtain horizontal collinations of $40^{\prime}-40^{\prime}-40^{\prime}-40^{\prime}$.

The c-axis sinusoidally modulated structure of $\mathrm{Tm}$ is characterized by a waverector $\vec{Q} \approx(0,0,2 / 7)$. The Nèel temperature of the specimen under study, measured as the temperature at which the first magnetic satellite at $\vec{\kappa}=(1,1,0) \pm \vec{Q}$ appears, was found to be $T_{. v}=58.5 \mathrm{~K}$. Most of the measurements were performed at $T=5 \mathrm{~K}$, a temperature at which $\mathrm{T} m$ orders in an antiphase-domain ferrimagnetic structure of characteristic wavevector $\vec{Q}=(0,0,2 / 7)$. At this temperature magnetic satellites are observed at $\vec{\kappa}=\vec{\tau} \pm \vec{Q}$ (where $\vec{\tau}$ is a reciprocal lattice vector) as well as at the higher harmonics $\vec{\kappa}=\vec{\tau} \pm(0,0,4 / 7)$ and $\vec{\kappa}=\vec{\tau} \pm(0,0,6 / 7) .^{4}$

The neutron scattering processes that are of interest in our study are: magnon creation, phonon creation (through neutron-nuclear interaction) and magneto-vibrational scattering (phonon creation through neutron-magnetic interaction). The neutron cross section for the creation of a magnon of wavevector $\vec{q}$ and energy $E_{q}$ in a periodic nagnetic structure of characteristic wavevector $\vec{Q}$ is ${ }^{1,6}$ :

$$
\begin{gathered}
\frac{d^{2} \sigma}{d \Omega d E_{M}}=\gamma(\vec{\kappa}) \frac{k_{f}}{k_{i}} \frac{1}{2 J}\left[n\left(E_{q}\right)+1\right] \sum_{\vec{r}}\left\{\frac{1}{4}\left(1+e_{Q}^{2}\right) A(\vec{q}) \delta(\vec{\kappa}-\vec{q} \pm \vec{Q}-\vec{\tau})\right. \\
\left.+\left(1-e_{Q}^{2}\right) B(\vec{q}) \delta(\vec{\kappa}-\vec{q}-\vec{\tau})\right\} \delta\left(E-E_{q}\right) .
\end{gathered}
$$

In this equation $\gamma(\vec{r})$ is a coefficient that contains the magnetic and geometrical form factors, and $A(\vec{q})$ and $B(\vec{q})$ are coefficients that depend on Fourier transforms of the exchange. The other symbols are those of standard usage: $k_{f}$ and $k_{i}$ are the final and initial wavevectors of the neutrons, $n\left(E_{q}\right)$ is the Bose thermal population factor, $\vec{e}$ is a unit vector in the direction of $\vec{\kappa}$ and $e_{Q}$ its component along $\vec{Q}$. Note from this cross section that an excitation of energy $E_{q}$ should be observed at $\vec{\kappa}=(\vec{q}+\vec{\tau})$ as well as at $\vec{r}=(\vec{q}+\vec{r} \pm \vec{Q})$ indicating that the magnon wavevector $\vec{q}$ is measured from a reciprocal lattice point $\vec{r}$ or from the magnetic satellites. The neutron cross section for magnetoribrational scattering in a periodic structure of characteristic waverector $\vec{Q}$ is ${ }^{6}$ :

$$
\begin{gathered}
\frac{d^{2} \sigma}{d \Omega d E_{. I V}}=\gamma(\vec{r}) \frac{k_{f}}{k_{i}}\left(\frac{J(T)}{J}\right)^{2}\left[n\left(E_{q}\right)+1\right] \frac{\hbar^{2} \kappa^{2}\left(\vec{e} \cdot \vec{\xi}_{q}\right)^{2}}{2 M E_{q}} \\
\times \sum_{\vec{r}} \frac{1}{2} \delta(\vec{r}-\vec{q} \pm \vec{Q}-\vec{\tau}) \delta\left(E-E_{q}\right) .
\end{gathered}
$$

Because this process is just the creation of a phonon through the neutron-magnetic interaction, its cross section is very similar to that for a phonon creation (through neutron-nuclear 
interaction): it is proportionl to $\kappa^{2}\left(\vec{e} \cdot \vec{\xi}_{q}\right)^{2} / E_{q}$, where $\vec{\xi}$ is the polarization vector of the phonon. Note, however, that unlike for the usual phonon case this cross section depends on the magnetic form factor, which is included in $\gamma(\vec{r})$, and on the magnetization. Also unlike the phonon case the waverector $\vec{q}$ is measured from the magnetic satellites.

The above cross sections indicate that in a neutron scattering experiment we should expect to observe a variety of branches in the dispersion relation: phonon and magnon branches from the reciprocal lattice points, and magnon and magnetoribrational scattering branches from each magnetic satellite.

\section{RESULTS AND DISCUSSION}

The low-temperature $(T=5 K)$ excitation spectra for $\mathrm{Tm}$ at all wavevectors under study consisted of three peaks. This can be seen in figure 1 , which shows constant- $\vec{r}$ scans at $\vec{r}=(1,1, \zeta)$ for $\zeta=0,0.3,0.8$. The solid lines in this figure are the result of leastsquares fits to three Gaussians. The excitation spectra along the $(0,0,2+\zeta)$ direction were qualitatively similar to those of figure 1 but the two lower-energy excitations were weaker. These spectra are also in qualitative agreement with those reported by McEwen and Steigenberger ${ }^{2}$ in the $0-12 \mathrm{meV}$ range. We found, however, no evidence of the excitation at $15 \mathrm{meV}$ reported by these authors.

In all cases the two lower-energy excitations are sharp and show a significant dispersion. The higher-energy excitation, on the other hand, exhibits only a weak dispersion (between 8.3 and $9.6 \mathrm{meV}$ ) and a linewidth that is waverector dependent. In figure 2 all the excitation energies identified from scans along the $(1,1, \zeta)$ and $(0,0,2+\zeta)$ directions have been plotted vs. $\zeta$. In this figure we have also plotted (in solid lines) the expected dispersion relation for the TA phonon (branch from $\zeta=0$ ) as well as the dispersion relations for magnetovibrational scattering from the TA phonon (branches from the magnetic satellites at $\zeta= \pm 2 / 7,-4 / 7$, the branches for $\zeta=4 / 7, \pm 6 / 7$ have been omitted). Because no phonon dispersion data are available for $\mathrm{Tm}$ the dispersion relation shown in this figure is that for $\mathrm{Tb}$ (of comparable mass and same structure) reported by Houmann and Nicklow. ${ }^{7}$ The dispersion relations for the magnetovibrational scattering shown in the same figure have been obtained by translating the phonon dispersion relation from $\zeta=0$ to $\zeta= \pm 2 / 7,-4 / 7$. The striking agreement of the lower-energy excitations and the solid lines strongly suggests that these should be identified as a TA phonon and magnetovibrational scattering from the TA phonon. We must remark that in principle the TA phonon should not be observed along the $(0,0,2+\zeta)$ direction due to the $\left(\vec{e} \cdot \vec{\xi}_{q}\right)^{2}$ dependence of the cross section of equation (3). In practice, however, it is not unusual that transverse phonons are observed in purely longitudinal scans due to multiple scattering processes (i.e. the creation of a phonon followed or preceded by Bragg scattering). The intensities of these lower-energy excitations along the $(1,1, \zeta)$ direction are consistent with the $\kappa^{2}\left(\vec{e} \cdot \vec{\xi}_{q}\right)^{2} / E_{q}$ dependence of the cross section of equation (3). Also the temperature dependence of these excitations, observed by McEwen and Steigenberger ${ }^{2}$ and ourselves, seems to be consistent with this cross section: the excitations that we identify as mag tovibrational scattering depend on the magnetization, which becomes smaller at higher :emperatures and vanishes at the Nècl temperature.

These observations suggest that only the higher-energy branch shown in figure 2 corresponds to magnetic excitations. A recent neutron scattering experiment performed with 
polarization analysis has confirmed this interpretation. ${ }^{8}$

The limited dispersion of this branch suggests thai : the Haniltonian is dominant over the exchange, and that the observed gap in the dispersion relation $(\approx 8 \mathrm{meV})$ may be closely related to the first dipolar transition in the crystalfield-only levels scheme. The energy of this transition can be estimated using the crystalfield paraneters estimated by Touborg ${ }^{9}$ from paramagnetic susceptibility measurements on dilute $\operatorname{Tm}$ alloys and pure Tm. This value is $5.5 \mathrm{meV}$ (from the dilute alloys estimations) or $7.7 \mathrm{meV}$ (from the pure Tm estimations).

Because these excitations are "magnons" (or magnon-like) the cross section of equation (2) suggests that several branches of the dispersion relation should be observed (from the reciprocal lattice point and from each satellite, like in the case of the magnetovibrational scattering discussed above). If such branches exist we have not been able to resolve them due to our relatively roarse energy resolution $\left(\Delta E_{F W H M}=1.4 \mathrm{meV}\right.$ at $\left.8 \mathrm{meV}\right)$. The fact that these excitations are broader than the energy resolution and that their linewidths are warevector dependent, on the other hand, suggests that such an interpretation of the data is not unreasonable. Upon warming these magnetic modes soften and become broader. These broad modes persist at least up to $T=70 \mathrm{~K}$. Further measurements are in progress.

\section{ACKNOWLEDGMENTS}

Work at ORNL supported by U.S. DOE under contract DE-AC05-84OR21400 with Martin Marietta Energy Systems, Inc. 


\section{REFEREICES}

[1] A. R. Machintosh and H. Bjerrum Moller, Spin Waves, in Magnetic Properties of Rare Earth Metals, edited by R. J. Elliott (Plenum Press, New York, 1972), chapter $\dot{ }$.

[2] K. A. McEwen and U. Steigenberger, J. de Physique 49, C8-335 (19S\$).

[3] J. A. Fernandez-Baca, R. M. Nicklow and J. J. Rhyne, Bull. Am. Phys. Soc. 34, 607 (1989).

[4] W. C. Koehler, J. W. Cable, E. O. Wollan, and M. K. Wilkinson, Phys. Rev. 126, 1672 (1962).

[5] P. A. Lingård, Phys. Rev. Lett. 36, 385 (1976); P. A. Lingård and J. F. Cooke, Phys. Rev. B14, 5056 (1976).

[6] H. Bjerrum Møller in Investigation of Magnons in Rare Earth Metals by Inelastic Neutron Scattering, Risö Report No. 178 (1968).

[7] J. C. G. Houmann and R. M. Nicklow, Phys. Rev. B1, 3943 (1970).

[S] J. A. Fernandez-Baca, R. M. Nicklow, Z. Tun and J. J. Rhyne, to be published.

[9] P. Touborg, Phys. Rev B16, 1201 (1977). 


\section{FIGURE CAPTIONS}

FIGURE 1. Constant $\vec{\kappa}$ scans at $\vec{\kappa}=(1,1, \zeta)(\zeta=0,0.3,0.8)$ for $\operatorname{Tm}$ at $T=5 K^{\circ}$. In all cases three excitations were observed, the lower energy ones show significant dispersion while the higher energy one exhibits only a weak dispersion. The solid lines are from a fit to three gaussians.

FIGURE 2. Dispersion relations of the observed excitations in $\operatorname{Tm}$ along the $(1,1, \zeta)$ (open circles) and $(0,0,2+\zeta)$ (full squares) directions. The solid lines correspond to the dispersion relation expected for the creation of a TA phonon (through nuclear interaction) as well as for magneto-vibrational scattering from the T.A phonon. 


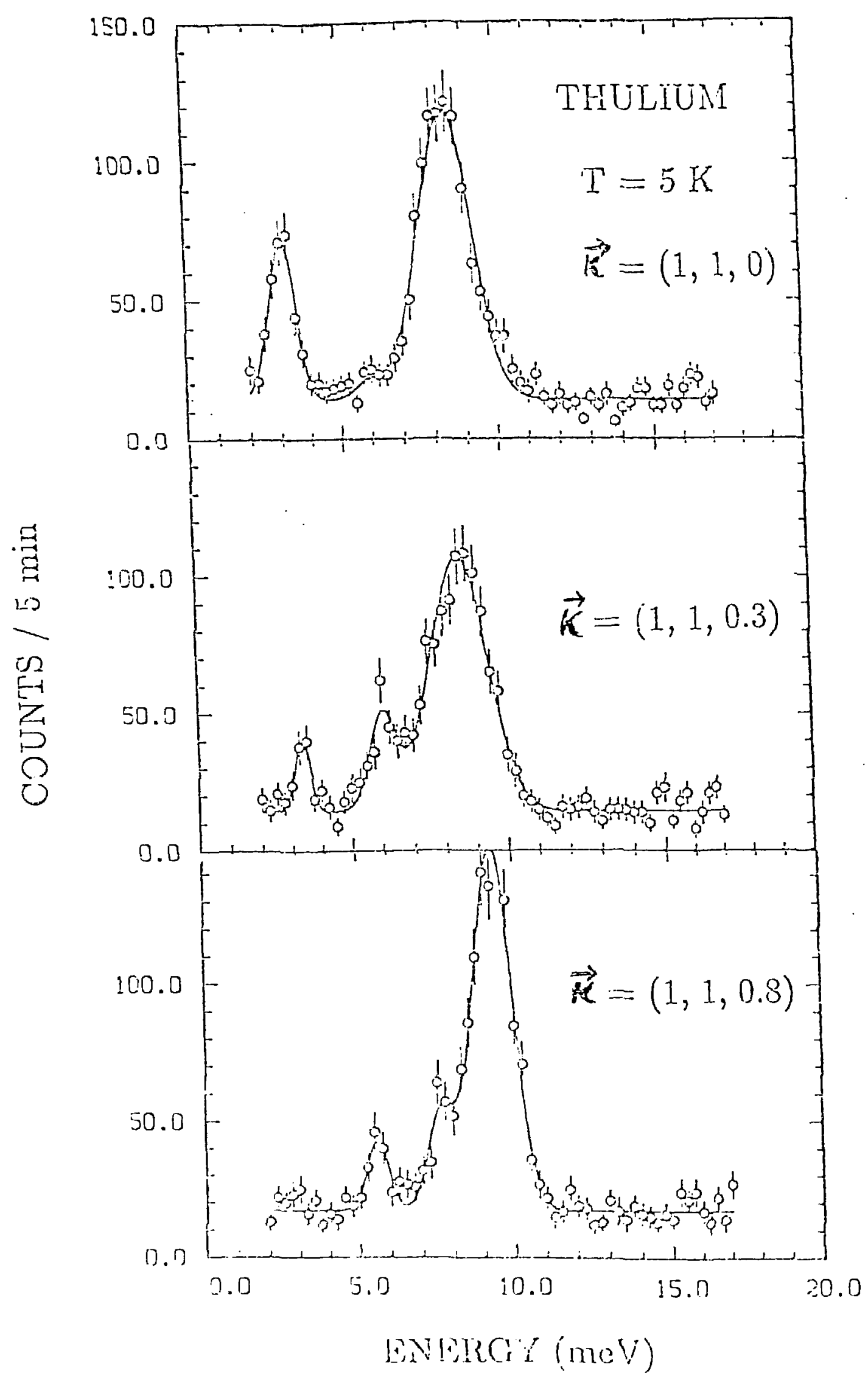




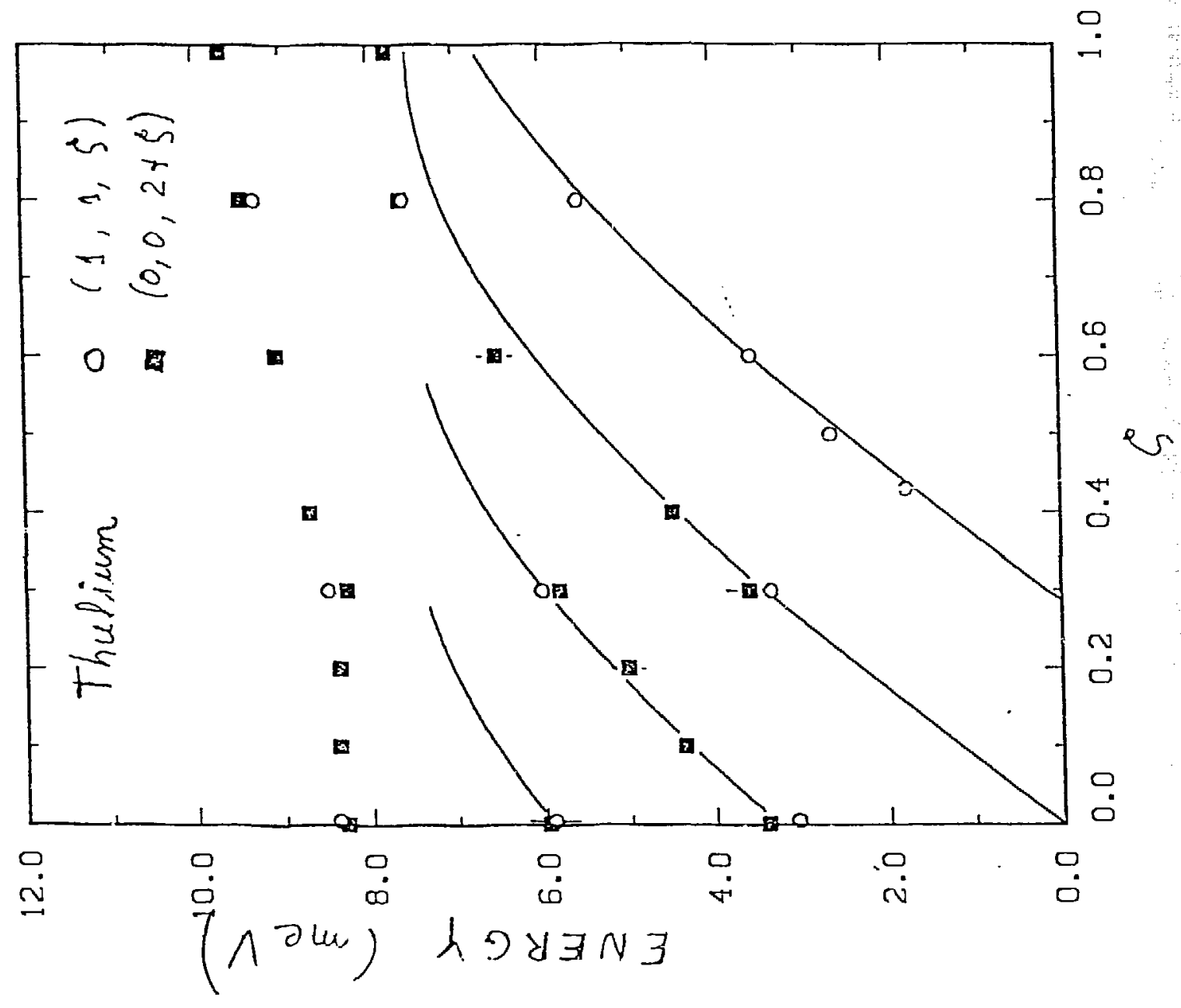

